\title{
Cardiac Power Is the Strongest Hemodynamic Correlate of Mortality in Cardiogenic Shock: A Report From the SHOCK Trial Registry
}

\author{
Rupert Fincke, MD,* Judith S. Hochman, MD, FACC, $†$ April M. Lowe, MS,
}

Venu Menon, MD, FACC, § James N. Slater, MD, FACC, † John G. Webb, MD, FACC, ,

Thierry H. LeJemtel, MD, FACC, 9 Gad Cotter, MD, FACC,\# for the SHOCK Investigators

New York and Bronx, New York; Watertown, Massachusetts; Chapel Hill and Durham, North Carolina; and Vancouver, Canada

\begin{abstract}
OBJECTIVES We sought to analyze clinical, angiographic, and outcome correlates of hemodynamic parameters in cardiogenic shock.

BACKGROUND The significance of right heart catheterization in critically ill patients is controversial, despite the prognostic importance of the derived measurements. Cardiac power is a novel hemodynamic parameter.

METHODS A total of 541 patients with cardiogenic shock who were enrolled in the SHould we emergently revascularize Occluded Coronaries for cardiogenic shocK (SHOCK) trial registry were included. Cardiac power output (CPO) (W) was calculated as mean arterial pressure $\times$ cardiac output/451.

RESULTS On univariate analysis, CPO, cardiac power index (CPI), cardiac output, cardiac index, stroke volume, left ventricular work, left ventricular work index, stroke work, mean arterial pressure, systolic and diastolic blood pressure (all $\mathrm{p}<0.001)$, coronary perfusion pressure $(\mathrm{p}=0.002)$, ejection fraction $(p=0.013)$, and pulmonary artery systolic pressure $(p=0.047)$ were associated with in-hospital mortality. In separate multivariate analyses, CPO (odds ratio per $0.20 \mathrm{~W}: 0.60$ [95\% confidence interval, 0.44 to 0.83 ], $\mathrm{p}=0.002 ; \mathrm{n}=181$ ) and CPI (odds ratio per $0.10 \mathrm{~W} / \mathrm{m}^{2}$ : 0.65 [95\% confidence interval, 0.48 to 0.87 ], $\mathrm{p}=0.004 ; \mathrm{n}=178$ ) remained the strongest independent hemodynamic correlates of in-hospital mortality after adjusting for age and history of hypertension. There was an inverse correlation between CPI and age (correlation coefficient: $-0.334, \mathrm{p}<0.001$ ). Women had a lower CPI than men $\left(0.29 \pm 0.11\right.$ vs. $\left.0.35 \pm 0.15 \mathrm{~W} / \mathrm{m}^{2}, \mathrm{p}=0.005\right)$. After adjusting for age, female gender remained associated with CPI $(p=0.032)$.

CONCLUSIONS Cardiac power is the strongest independent hemodynamic correlate of in-hospital mortality in patients with cardiogenic shock. Increasing age and female gender are independently associated with lower cardiac power. (J Am Coll Cardiol 2004;44:340-8) (c) 2004 by the American College of Cardiology Foundation
\end{abstract}

The usefulness of hemodynamic measurements with right heart catheterization (RHC) in critically ill patients has been questioned (1,2). Echocardiography has largely obviated the role of RHC as a diagnostic tool for the etiology of cardiogenic shock (3-5), and the echocardiographic assessment of cardiac function is of prognostic importance $(6,7)$. Although studies examining the utility of RHC and measurement of pulmonary capillary wedge pressure (PCWP) and cardiac output $(\mathrm{CO})$ did not demonstrate significant beneficial effects on patient outcome $(1,8,9)$, PCWP and $\mathrm{CO}$ had independent prognostic value in 995 patients in

From the *New York Presbyterian Hospital-Weill Cornell Medical Center, New York, New York; †New York University School of Medicine, New York, New York; ‡New England Research Institutes, Watertown, Massachusetts; §University of North Carolina, Chapel Hill, North Carolina; |St. Paul's Hospital, Vancouver, Canada; IAlbert Einstein College of Medicine, Bronx, New York; and \#Duke University Medical Center, Durham, North Carolina. Supported by R01 grants HL50020, HL49970 from the National Heart, Lung, and Blood Institute, National Institutes of Health, Bethesda, Maryland.

Manuscript received December 11, 2003; revised manuscript received March 8, 2004, accepted March 16, 2004. cardiogenic shock in the Global Utilization of Streptokinase and Tissue Plasminogen Activator for Occluded Coronary Arteries-I (GUSTO-I) trial (10).

The "SHould we emergently revascularize Occluded Coronaries for cardiogenic shocK" (SHOCK) trial was a randomized trial of a strategy of early revascularization for cardiogenic shock complicating an acute myocardial infarction (MI) (11). The results of the randomized trial have been reported (12). Patients who were ineligible for the randomized trial or who were trial-eligible but not randomized were prospectively enrolled in the SHOCK trial registry.

The purpose of the present study was to evaluate clinical, angiographic, and outcome correlates of hemodynamic parameters of patients with suspected cardiogenic shock secondary to left ventricular (LV) dysfunction after an acute MI.

In addition to analyzing traditional hemodynamic variables including blood pressure, PCWP, cardiac index (CI), and systemic vascular resistance (SVR), we evaluated a novel hemodynamic measure: cardiac power. Using the physical 


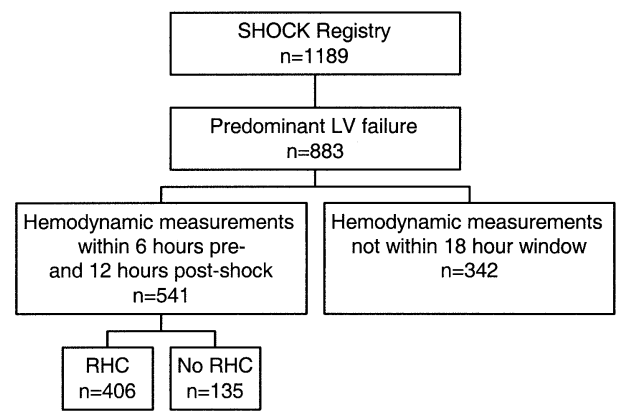

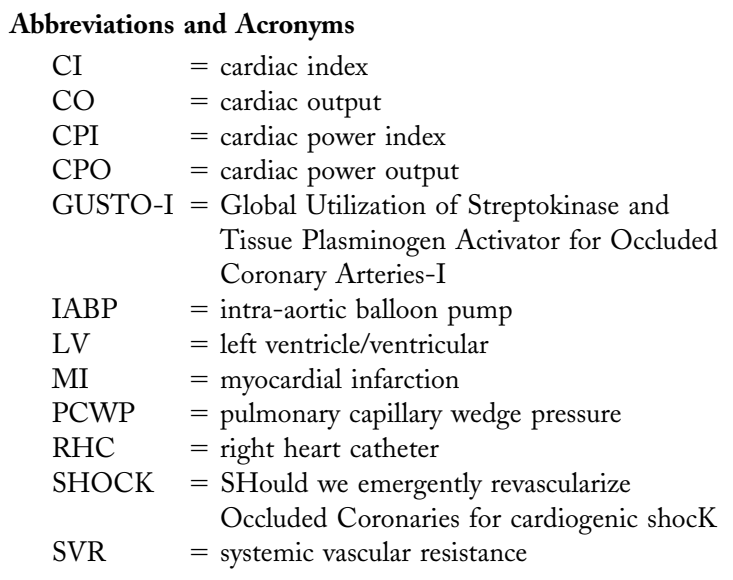

rule of fluids (power $=$ pressure $\times$ flow), cardiac power is the product of simultaneously measured cardiac output (or index) and mean arterial blood pressure. Therefore, by coupling both pressure and flow domains of the cardiovascular system, it is a measure of cardiac pumping (13). Cardiac power can be determined without RHC noninvasively using various devices (14). In recent studies, cardiac power was shown to be instrumental in the diagnosis of cardiogenic shock (15) and an important determinant of outcome in patients with cardiogenic shock (16) and chronic heart failure (17).

\section{METHODS}

Study design and data collection. The design of the randomized SHOCK trial and SHOCK trial registry has been published $(11,18)$. Enrollment in the SHOCK trial registry required only that cardiogenic shock was suspected on clinical grounds; RHC was not mandated. Patients were prospectively registered at 36 international sites between April 1993 and August 1997. Data were collected from patient charts by centrally trained study coordinators who entered the data onto standardized study report forms. Patient characteristics, MI characteristics, hemodynamic data, medications, procedures used, and vital status at discharge were recorded. The coronary angiograms obtained in the SHOCK trial registry were analyzed by the local site investigators. Data from cardiac catheterization reports from all sites were abstracted at the SHOCK trial clinical coordinating center where standardized report forms were completed.

Definitions. Predominant LV failure was indicated by the site investigators as the etiology of cardiogenic shock if none of the following shock categories was thought to be present: isolated right ventricular shock, acute severe mitral regurgitation, ventricular septal rupture, cardiac tamponade or rupture, prior severe valvular heart disease, dilated cardiomyopathy, excess beta or calcium channel blockade, and cardiogenic shock associated with recent hemorrhage or cardiac catheterization laboratory complication.
Figure 1. Time of hemodynamic measurements and right heart catheter (RHC) status of patients with left ventricular (LV) failure in the SHould we emergently revascularize Occluded Coronaries for cardiogenic shocK (SHOCK) registry.

Patient population and hemodynamic measurements. The following report is based on 541 patients with predominant LV failure whose hemodynamic measurements were made between $6 \mathrm{~h}$ before and up to $12 \mathrm{~h}$ after shock diagnosis (Fig. 1). Measurements of LV ejection fraction were included if they were obtained one day before, on the same day as, or one day after shock diagnosis.

Of the 541 patients analyzed, 406 (75\%) underwent RHC (Fig. 1). Compared with patients who did not undergo RHC, those with RHC were younger; less likely to have had a prior MI or renal insufficiency; had higher systolic blood pressure and diastolic blood pressure, heart rate, and peak CPK; and were more frequently treated with dobutamine and an intra-aortic balloon pump (IABP) (Table 1).

The following measures were available on only one-half to approximately two-thirds of the patients undergoing RHC due to an expansion of the case report forms during the study period: $\mathrm{CO}$, mean right atrial pressure, pulmonary artery systolic pressure, and pulmonary artery diastolic pressure. In addition, right ventricular systolic pressure and right ventricular diastolic pressure were optional and only available on just over one-fifth of RHC patients. Cardiac index was recorded in 282 patients and PCWP recorded in 378. Left ventricular ejection fraction was measured in 196 patients (in $52 \%$ by echocardiography, in $47 \%$ by $\mathrm{LV}$ angiography, and in $0.5 \%$ by gated blood pool scan).

An estimate of coronary perfusion pressure was calculated as diastolic blood pressure - PCWP. Nine patients with a coronary perfusion pressure $\leq 0 \mathrm{~mm} \mathrm{Hg}$ were excluded from this analysis $(n=329)$. Their coronary perfusion pressure results are likely due to nonsimultaneous measurements of diastolic blood pressure and PCWP or technical problems (underestimation of diastolic blood pressure with arm cuff, zero position for RHC at bedside).

Cardiac power output (CPO) (W) was calculated as mean arterial pressure $\times \mathrm{CO} / 451$, where mean arterial pressure $=$ [(systolic blood pressure - diastolic blood pressure)/3] + diastolic blood pressure. Systemic vascular resistance (dyne $\mathrm{s} / \mathrm{cm}^{5}$ ) was determined by the following equation: $80 \times$ (mean arterial pressure - right atrial pressure)/CO. Cardiac 
Table 1. Baseline Demographic and Clinical Characteristics of Patients With Shock Due to Predominant Left Ventricular Failure Overall and by Right Heart Catheterization

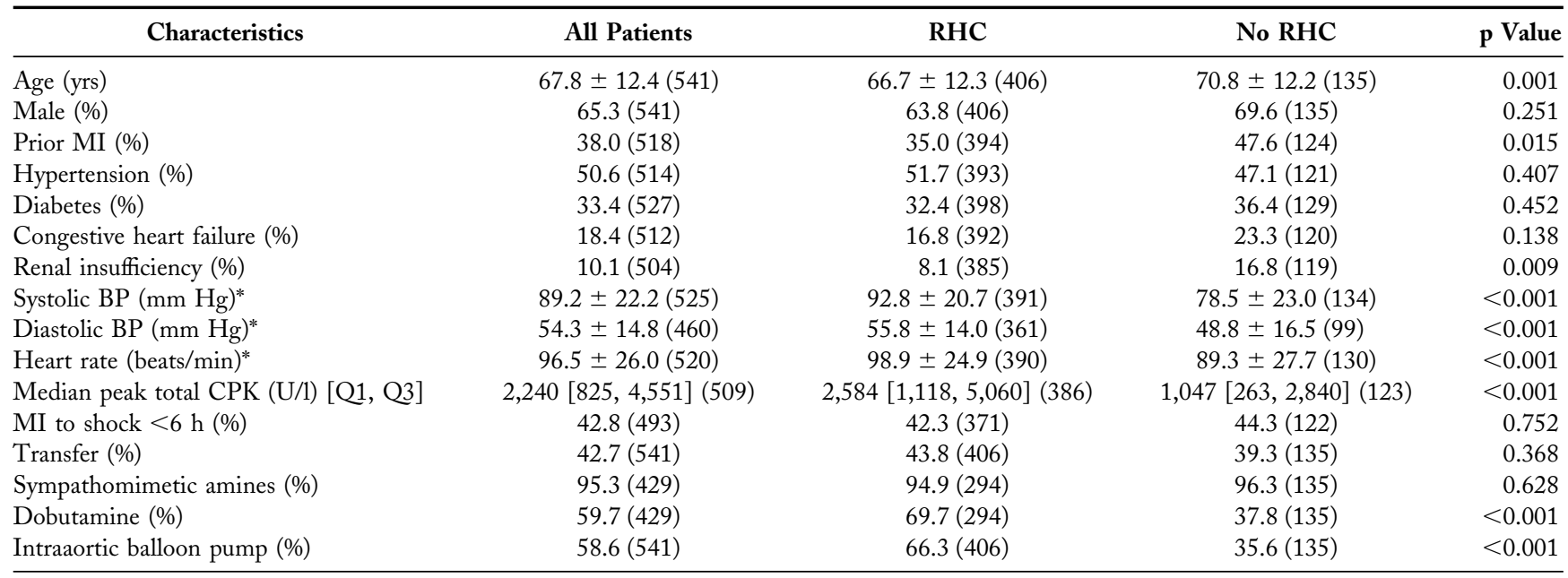

*Measurements were obtained while on inotropic, sympathomimetic amine, and/or intraaortic balloon pump support. ( ) = number of patients with available data; [ ] $=25$ th-75th percentile.

$\mathrm{BP}=$ blood pressure $\mathrm{CPK}=$ creatine phosphokinase $\mathrm{MI}=$ myocardial infarction; $\mathrm{RHC}=$ right heart catheter

power index $(\mathrm{CPI})\left(\mathrm{W} / \mathrm{m}^{2}\right)$ and systemic vascular resistance index (dyne $\mathrm{s} / \mathrm{cm}^{5} / \mathrm{m}^{2}$ ) were computed by substituting CO with $\mathrm{CI}$ in the respective formulas. One patient with a negative value for SVR was excluded from any analyses concerning SVR and SVR index. Stroke work (g-m) was calculated as (mean arterial pressure $-\mathrm{PCWP}) \times 0.0136 \times$ stroke volume. Stroke work index $\left(\mathrm{g}-\mathrm{m} / \mathrm{m}^{2}\right)$ was determined by stroke work/body surface area. Left ventricular work was defined as (mean arterial pressure $-\mathrm{PCWP}) \times \mathrm{CO} \times$ $0.0136(\mathrm{~kg}-\mathrm{m} / \mathrm{min})$. Left ventricular work index was calculated as (mean arterial pressure $-\mathrm{PCWP}) \times \mathrm{CI} \times 0.0136$ $\left(\mathrm{kg}-\mathrm{m} / \mathrm{min} \times \mathrm{m}^{2}\right)$. Hemodynamic measurements included those recorded while the patients were receiving supportive therapy (sympathomimetic amines, 95\%; and/or dobutamine, 60\%; and/or IABP, 59\%). If a patient had no IABP or if the RHC measurements were obtained before insertion of the IABP or after removal of the IABP, then RHC data were considered off IABP (73\%). Right heart catheter measurements made in the time interval between IABP insertion and removal were considered on IABP support (27\%).

Statistical methods. Descriptive statistics are presented as mean $\pm \mathrm{SD}$ for continuous data (or median and quartiles for skewed data) and percentages for categorical data. A $p$ value of $\leq 0.05$ was considered as statistically significant. Fisher's exact test was used to compare all group proportions.

Associations of continuous variables with patient characteristic subgroups such as right heart catheterization, gender, history of hypertension, prior MI, history of congestive heart failure, diabetes, and renal insufficiency were examined using Student's $t$ test for normally distributed variables (heart rate) and the Wilcoxon rank-sum test for nonnormally distributed variables.

Hemodynamic parameters by culprit artery and number of diseased vessels were analyzed using analysis of variance to compare normally distributed variables (heart rate and pulmonary artery diastolic pressure) and the Kruskal-Wallis test to compare non-normally distributed variables. For significant overall effects, pairwise comparisons used the Bonferroni method of adjustment to determine significance.

Logistic regression analysis was used to examine the relationship between in-hospital mortality and hemodynamic parameters and to display predicted mortality as a function of CPO. Units for odds ratios from logistic regression were determined using a rule of approximately $50 \%$ of the SD with rounding to the next appropriate unit.

Using logistic regression and linear regression, patient characteristic and hemodynamic correlates of $\mathrm{CPO}$ or CPI and in-hospital mortality were assessed. In the subset of patients with CPO data, logistic regression was performed to determine correlates of both CPI and in-hospital mortality. Those variables that remained significant at the 0.20 level when modeled with CPI were entered into a multivariate model. Single variables were removed in a stepwise method until a model meeting a 0.05 significance level in all variables was attained. These steps were repeated with CPO.

In addition, receiver operator characteristic analysis was performed to determine a cutpoint for $\mathrm{CPO}$ that provides an approximately equivalent sensitivity and specificity for predicting in-hospital mortality.

Linear regression, with log-transformation of CI to achieve normality, was used to study the relationship between CI, CPI, SVR, SVR index, including age, gender, and hemodynamics.

Spearman correlation coefficients were used to estimate univariate associations between CI, CPI, or SVR, and age.

Analyses were conducted using the Statistical Analysis System (SAS, version 8.2., Cary, North Carolina) and S-Plus 6 (Insightful Corp., Seattle, Washington) software. 
Table 2. Univariate Logistic Regression Results of Hemodynamic Parameters for In-Hospital Mortality

\begin{tabular}{|c|c|c|c|c|c|}
\hline Parameters* & $\mathbf{n}$ & Mean $\pm \mathrm{SD} \dagger$ & $\begin{array}{l}\text { Unit for } \\
\text { Odds Ratio }\end{array}$ & $\begin{array}{c}\text { Odds Ratio } \\
\text { (95\% CI) }\end{array}$ & p Value \\
\hline Cardiac power output & 189 & $0.62 \pm 0.30$ & 0.20 & $0.55(0.41,0.73)$ & $<0.001$ \\
\hline Cardiac power index & 253 & $0.33 \pm 0.14$ & 0.10 & $0.61(0.49,0.77)$ & $<0.001$ \\
\hline Stroke volume & 196 & $40.8 \pm 18.5$ & 10 & $0.69(0.56,0.84)$ & $<0.001$ \\
\hline Left ventricular work index & 244 & $1.36 \pm 0.74$ & 0.4 & $0.69(0.58,0.82)$ & $<0.001$ \\
\hline Left ventricular work & 182 & $2.61 \pm 1.54$ & 0.7 & $0.70(0.58,0.84)$ & $<0.001$ \\
\hline Stroke work index & 154 & $14.8 \pm 9.1$ & 5 & $0.72(0.58,0.91)$ & 0.005 \\
\hline Cardiac index & 282 & $2.07 \pm 0.79$ & 0.5 & $0.73(0.62,0.87)$ & $<0.001$ \\
\hline Left ventricular ejection fraction & 196 & $30.0 \pm 12.8$ & 7 & $0.81(0.69,0.96)$ & 0.013 \\
\hline Systolic blood pressure & 525 & $89.2 \pm 22.2$ & 10 & $0.84(0.77,0.92)$ & $<0.001$ \\
\hline Mean arterial pressure & 460 & $66.7 \pm 15.9$ & 8 & $0.84(0.76,0.93)$ & $<0.001$ \\
\hline Coronary perfusion pressure & 329 & $33.4 \pm 14.4$ & 7 & $0.84(0.75,0.94)$ & 0.002 \\
\hline Pulmonary artery diastolic pressure & 260 & $23.7 \pm 7.4$ & 4 & $1.10(0.96,1.26)$ & 0.164 \\
\hline Pulmonary artery systolic pressure & 259 & $40.3 \pm 11.8$ & 6 & $1.14(1.00,1.29)$ & 0.047 \\
\hline SVR index & 144 & $2,266[1,782,2,860] \dagger$ & 600 & $1.14(0.93,1.39)$ & 0.203 \\
\hline SVR & 143 & $1,257[938,1,620] \dagger$ & 400 & $1.16(0.93,1.44)$ & 0.186 \\
\hline Right ventricular systolic pressure & 93 & $43.7 \pm 15.8$ & 8 & $1.17(0.94,1.46)$ & 0.161 \\
\hline
\end{tabular}

${ }^{*}$ Measurements were obtained while on inotropic, sympathomimetic amine, and/or intra-aortic balloon pump support. $†$ Median given for SVR and SVR index. [ ] $=25$ th-75th percentile.

$\mathrm{CI}=$ confidence interval; PCWP $=$ pulmonary capillary wedge pressure; SVR $=$ systemic vascular resistance.

\section{RESULTS}

Hemodynamic correlates of in-hospital mortality. The overall mortality of the examined population $(n=541)$ was $56.8 \%$. The mortality of the subset of patients with CPO data $(\mathrm{n}=189)$ was significantly lower than those without CPO data $(\mathrm{n}=352)(42.9 \%$ vs. $64.2 \%, \mathrm{p}<0.001)$.

On univariate analysis, a number of hemodynamic variables were correlated with in-hospital mortality (Table 2). Multivariate logistic regression models were used to assess the strength of CPI $(n=178)$ and CPO $(n=181)$ as correlates of in-hospital mortality after adjusting for patient characteristics and/or other hemodynamic parameters related to both in-hospital mortality and CPI or CPO. Cardiac power index $(\mathrm{p}=0.004)$ and $\mathrm{CPO}(\mathrm{p}=0.002)$ remained independently associated with in-hospital mortality after adjusting for the only remaining significant covariates, age and history of hypertension (Tables 3 and 4). In addition, after adjusting for age and hypertension, $L V$ work index $(\mathrm{n}=171)$ with an odds ratio $(95 \%$ confidence interval) per $0.4 \mathrm{~kg}-\mathrm{m} / \mathrm{min} \times \mathrm{m}^{2}$ increase of $0.75(0.60$,

Table 3. Multivariate Logistic Regression Results for In-Hospital Mortality (Using Cardiac Power Index) $(\mathrm{n}=178)$

\begin{tabular}{lcr}
\hline \multicolumn{1}{c}{ Variables } & $\begin{array}{c}\text { Odds Ratio } \\
\mathbf{( 9 5 \%} \text { Confidence Interval) }\end{array}$ & \multicolumn{1}{c}{$\begin{array}{c}\mathbf{p} \\
\text { Value }\end{array}$} \\
\hline Cardiac power index & $0.65(0.48,0.87)^{*}$ & 0.004 \\
Age & $1.78(1.31,2.42)^{*}$ & $<0.001$ \\
History of hypertension & $0.46(0.22,0.94)$ & 0.033 \\
\hline
\end{tabular}

*The odds ratio and confidence interval for cardiac power index is per $0.10 \mathrm{~W} / \mathrm{m}^{2}$ increase. The odds ratio and confidence interval for age is per 10-year increase.
$0.93), \mathrm{p}=0.009$, and $\mathrm{LV}$ work $(\mathrm{n}=174)$ with an odds ratio ( $95 \%$ confidence interval) per $0.7 \mathrm{~kg}-\mathrm{m} / \mathrm{min}$ increase of 0.75 (0.60, 0.92), $\mathrm{p}=0.006$, also remained independent, but weaker, correlates of in-hospital mortality. Cardiac power index, CPO, LV work index, and LV work continued to be significant correlates of mortality after controlling for IABP use. Predicted in-hospital mortality as a function of CPO with pointwise 95\% confidence bands is displayed in Figure 2. The odds ratio for mortality without adjustment for baseline characteristics is 0.55 for each $0.20-\mathrm{W}$ increase in $\mathrm{CPO}$, that is, there is a $45 \%$ decrease in the odds of death.

Cutoff value for CPO to determine in-hospital mortality. Because $\mathrm{CPO}$ was the strongest independent hemodynamic correlate of outcome in the present cohort, we have attempted to determine a cutoff value for CPO that is most accurately related to outcome. By receiver operator characteristic analysis, assuming an approximately equal sensitivity and specificity of 0.66 , a CPO of $0.53 \mathrm{~W}$ was found to most accurately predict in-hospital mortality (c-statistic $=0.69$ ). The probability of in-hospital mortality with a $\mathrm{CPO} \leq 0.53$

Table 4. Multivariate Logistic Regression Results for In-Hospital Mortality (Using Cardiac Power Output) $(\mathrm{n}=181)$

\begin{tabular}{lcr}
\hline \multicolumn{1}{c}{ Variables } & $\begin{array}{c}\text { Odds Ratio } \\
\text { (95\% Confidence Interval) }\end{array}$ & \multicolumn{1}{c}{$\begin{array}{c}\mathbf{p} \\
\text { Value }\end{array}$} \\
\hline Cardiac power output & $0.60(0.44,0.83)^{*}$ & 0.002 \\
Age & $1.75(1.29,2.38)^{*}$ & $<0.001$ \\
History of hypertension & $0.46(0.23,0.94)$ & 0.033 \\
\hline *The odds ratio and confidence interval for cardiac power output is per $0.20 \mathrm{~W}$ \\
increase. The odds ratio and confidence interval for age is per 10-year increase.
\end{tabular}




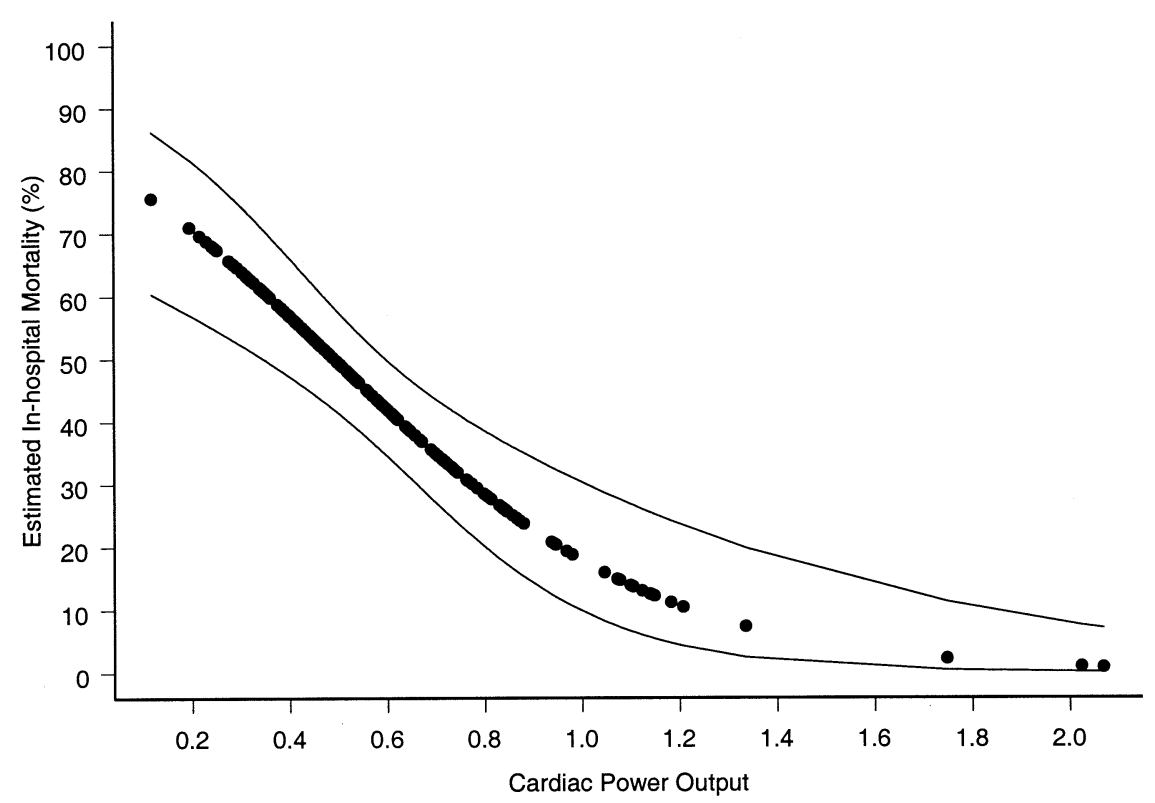

Figure 2. Unadjusted estimated in-hospital mortality by cardiac power output $(\mathrm{n}=189)$ with pointwise $95 \%$ confidence bands.

W was $58 \%$ (positive predictive value), whereas the probability of survival given a $\mathrm{CPO}>0.53 \mathrm{~W}$ was $71 \%$ (negative predictive value).

Hemodynamics in relation to baseline characteristics. We found a significant inverse correlation between CPI and age (correlation coefficient, $-0.334, \mathrm{p}<0.001$ ), whereas age and SVR were positively correlated $(0.347, \mathrm{p}<0.001)$.

Women had a significantly lower CPI and CI than men $\left(0.29 \pm 0.11\right.$ vs. $0.35 \pm 0.15 \mathrm{~W} / \mathrm{m}^{2}, \mathrm{p}=0.005$ and $1.82 \pm$ 0.57 vs. $\left.2.22 \pm 0.87 \mathrm{1} / \mathrm{min} / \mathrm{m}^{2}, \mathrm{p}<0.001\right)$ and a higher median SVR (1,456 [interquartile range, $1,248,2,016$ ] vs. $1,095[801,1,360]$ dyne $\left.\mathrm{s} / \mathrm{cm}^{5}, \mathrm{p}<0.001\right)$. Although women were significantly older than men $(70.7 \pm 11.6$ vs. $66.2 \pm 12.6$ years, $\mathrm{p}<0.001)$, after adjusting for age, female gender remained associated with CPI $(p=0.032)$, CI $(p=0.002)$, and SVR $(p<0.001)$. In the subset of patients with SVR data $(\mathrm{n}=142)$, support with epinephrine $(42.9 \%$ vs. $26.7 \%, p=0.067)$ and norepinephrine ( $30.4 \%$ vs. $18.6 \%, p=0.154)$ tended to be used more frequently in women compared with men, although not statistically significant. Dopamine use was similar in women and men ( $87.5 \%$ vs. $86.1 \%, p=1.000)$. After adjusting for epinephrine and norepinephrine use, female gender remained a significant correlate of SVR (both, $\mathrm{p}<0.001$ ). Also, in the subset of patients with SVR data $(n=143)$, utilization of IABP support was comparable for women and men $(59.7 \%$ vs. $62.8 \%, p=0.728)$.

A total of $51 \%$ of patients had a history of hypertension, while $38 \%$ of patients had a prior MI, and $18 \%$ had congestive heart failure. A history of hypertension was associated with lower CPI $(0.31 \pm 0.15$ vs. $0.35 \pm 0.13$ $\left.\mathrm{W} / \mathrm{m}^{2}, \mathrm{p}<0.001\right)$, whereas a prior MI $(0.32 \pm 0.14$ vs. $\left.0.33 \pm 0.14 \mathrm{~W} / \mathrm{m}^{2}, \mathrm{p}=0.880\right)$ and a history of congestive heart failure $\left(0.30 \pm 0.10\right.$ vs. $0.33 \pm 0.15 \mathrm{~W} / \mathrm{m}^{2}, \mathrm{p}=$
0.596) were not. None of these conditions were associated with SVR. In contrast, mean PCWP was similar for patients with and without a history of hypertension (23.9 \pm 8.8 vs. $24.4 \pm 8.8 \mathrm{~mm} \mathrm{Hg}, \mathrm{p}=0.768$ ), whereas patients with a prior MI $(25.9 \pm 9.0$ vs. $23.2 \pm 8.5 \mathrm{~mm} \mathrm{Hg}, \mathrm{p}=$ $0.003)$ and with a history of congestive heart failure (26.8 \pm 11.3 vs. $23.5 \pm 8.1 \mathrm{~mm} \mathrm{Hg}, \mathrm{p}=0.016$ ) had significantly higher PCWP than patients without either such a history.

Diabetics comprised $33 \%$ of all patients, and $10 \%$ of all patients had renal insufficiency. Diabetics and nondiabetics had comparable hemodynamics (including CPI), except for median SVR, diastolic blood pressure, and coronary perfusion pressure, which were significantly lower in the diabetic patients (SVR 1,064 [844, 1,410] vs. 1,298 [1,037, 1,782] dyne $\mathrm{s} / \mathrm{cm}^{5}, \mathrm{p}=0.024$; diastolic blood pressure $52.1 \pm 14.2$ vs. $55.5 \pm 15.1 \mathrm{~mm} \mathrm{Hg}, \mathrm{p}=0.004$ and coronary perfusion pressure $30.5 \pm 13.5$ vs. $34.8 \pm 14.6 \mathrm{~mm} \mathrm{Hg}, \mathrm{p}=0.010$ ). Hemodynamic measures in patients with and without prior renal insufficiency were similar.

Hemodynamic measurements, culprit artery, and extent of coronary disease. Patients with left main artery related MI had higher pulmonary artery systolic pressures than patients with right coronary artery related MI $(53.4 \pm 12.5$ vs. $37.3 \pm 10.8 \mathrm{~mm} \mathrm{Hg}, \mathrm{p}=0.032)$ and higher PCWP than patients with left anterior descending related MI (33.5 \pm 11.2 vs. $23.4 \pm 9.4 \mathrm{~mm} \mathrm{Hg}, \mathrm{p}=0.017$ ) or right coronary artery related $\mathrm{MI}(33.5 \pm 11.2$ vs. $23.2 \pm 7.7 \mathrm{~mm} \mathrm{Hg}, \mathrm{p}=$ 0.011 ) (Table 5). The coronary perfusion pressure in patients with left main MI was lower than for all other culprit arteries $(26.0 \pm 21.9$ vs. $34.5 \pm 13.9 \mathrm{~mm} \mathrm{Hg}, \mathrm{p}=0.054)$.

Standard hemodynamic parameters including SVR, SVR index, and CPI were unrelated to the number of diseased vessels, whereas coronary perfusion pressure was significantly lower in patients with three-vessel disease compared 
Table 5. Selected Hemodynamic Parameters of Patients With Shock Due to Predominant Left Ventricular Failure by Culprit Artery

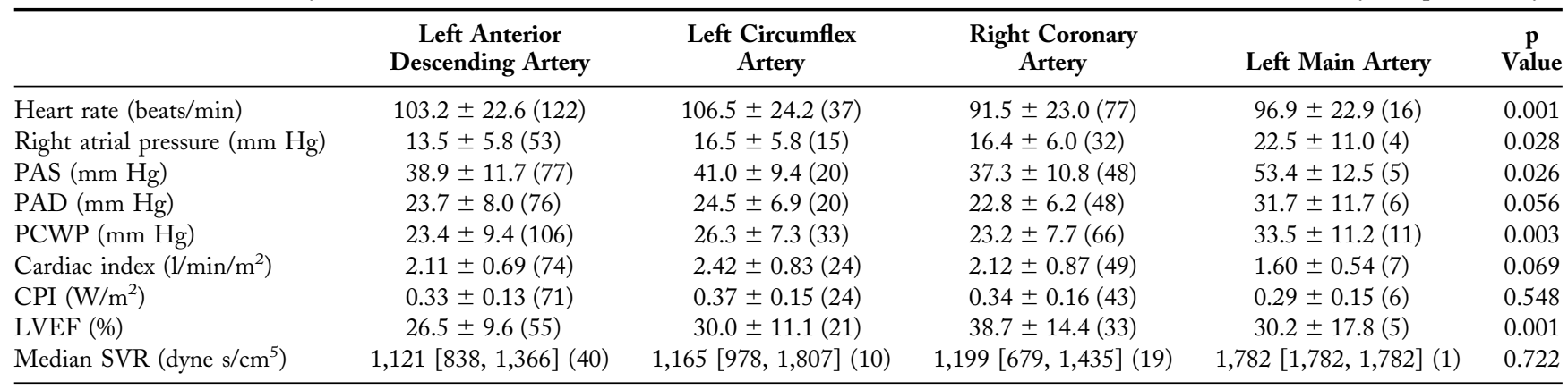

Measurements were obtained while on inotropic, sympathomimetic amine, and/or intraaortic balloon pump support. ( ) = number of patients with available measurements; [ ] = 25 th-75th percentile.

$\mathrm{CPI}=$ cardiac power index; LVEF = left ventricular ejection fraction; PAD = pulmonary artery diastolic pressure; PAS = pulmonary artery systolic pressure; PCWP = pulmonary capillary wedge pressure; SVR = systemic vascular resistance.

with patients with either zero/one-, or two-vessel disease by pairwise comparison $(31.5 \pm 14.5$ vs. $36.2 \pm 12.6 \mathrm{~mm} \mathrm{Hg}$, $\mathrm{p}=0.049$ or vs. $37.7 \pm 15.4 \mathrm{~mm} \mathrm{Hg}, \mathrm{p}=0.029)$.

\section{DISCUSSION}

Limitations of traditional hemodynamic measures. Hemodynamic monitoring has been used extensively during the last decades for risk stratification and guiding treatment of patients with cardiovascular destabilization, especially acute heart failure and cardiogenic shock. However, in recent years, RHC has been complemented by echocardiographic evaluation in the risk stratification of these patients (19). The echocardiographic measurement of LV ejection fraction and the severity of mitral regurgitation are strongly correlated with the outcome of patients in cardiogenic shock (20).

Although RHC-derived hemodynamic measurements like PCWP and CO are correlated with outcome (10), RHC use, per se, is not associated with any benefit $(1,2)$ and has raised concerns about harm $(1,2,8)$. This observation is likely responsible for the diminishing use of RHC in patients with hemodynamic instability (21), despite the lack of sufficiently powered randomized trials (22).

There are various possible explanations for this apparent paradox. Some of the current treatments applied to patients with acute heart failure and cardiogenic shock were shown to improve symptoms, but not final outcome. Hence, the titration of medications to achieve a set of predetermined hemodynamic goals might be actually detrimental to the patient (23-25). Moreover, it is possible that the hemodynamic measures and their presumed target values used in "treatment protocols" (26) are misleading. Primarily, CI and PCWP are currently used for hemodynamic monitoring. However, CI is not an index of cardiac contractility, but rather a measure of cardiovascular flow and, therefore, affected by contractility, vascular stiffness, and resistance, as well as intravascular volume and ventricular filling pressures. Pulmonary capillary wedge pressure is a measure of intracardiac pressure, reflecting ventricular compliance and volume status, but not directly representing cardiac performance or pumping ability. Therefore, although these measures are correlated with patient outcome in some studies, they might be only secondarily related to more fundamental hemodynamic effectors.

Cardiac power. In attempting to characterize a system composed of an energy source and pipes conducting this energy, the usual parameters used for characterizing the system are the power of the energy generator and the resistance of the conducting pipes. In the cardiovascular system, the pumping power of the heart can be determined by cardiac power, which is the product of simultaneously measured CO (cardiovascular flow) and mean arterial (=intravascular) pressure.

Tan et al. (16) has shown that the resting CPO for a hemodynamically stable average sized adult is approximately $1 \mathrm{~W}$. However, during stress or exercise, cardiac power reserve can be recruited to increase the heart's pumping ability up to $6 \mathrm{~W}$. In patients with chronic heart failure, this recruitable reserve is significantly diminished and is strongly correlated with outcome $(17,27)$.

In acute heart failure, on the other hand, the patient becomes hemodynamically unstable, and most of the cardiac pumping potential is recruited in order to sustain life. Hence, cardiac power measurements in patients with acute heart failure at rest represent most of the recruitable reserve available during the acute event, and their measurement reflects the severity of the patient's condition.

In a recent study (15), we evaluated hemodynamic variables in patients with acute hemodynamic decompensation due to a variety of causes. We observed that CI, PCWP, and blood pressure were not instrumental in determining the patient's exact diagnosis. It could be determined, however, with an accuracy of $95 \%$ by combining individual measures of cardiac power and SVR. Moreover, studies attempting to use CI and PCWP changes as measures for treatment modulation in heart failure failed to show any significant clinical effect (28). We were also able to show that cardiac power measured at admission in patients with acute heart failure is strongly correlated with the rate of recurrent heart failure events during follow-up (29).

The results of the present study demonstrate that many hemodynamic variables including mean arterial pressure, $\mathrm{CO}$, 
$\mathrm{CI}$, and $\mathrm{LV}$ ejection fraction are correlated with outcome in patients with cardiogenic shock secondary to severe LV dysfunction. In contrast with the findings in GUSTO-I, PCWP was not a correlate of mortality in our study. On multivariate analysis, cardiac power and LV work were found to be the only independent hemodynamic correlates of in-hospital mortality, with cardiac power being a stronger correlate than LV work. Cardiac power represents the rate of energy input the systemic vasculature receives from the heart at the level of the aortic root. This is the amount of energy available to maintain the perfusion of the vital organs in shock. Left ventricular cardiac work, in contrast, is the amount of energy imparted only by the $\mathrm{LV}$ to the volume of blood ejected per minute. It does not include the contribution of the left atrium to the maintenance of the systemic circulation. Also, CPO was a better correlate than CPI. Several studies have demonstrated an independent association between low body weight, short height, and increased mortality after an acute MI (30-32). Therefore, the adjustment of CPO for body size yielding CPI may result in a weaker association with mortality. Furthermore, body surface area may not be an adequate measure to correct for patient size and may skew the correlation of CPI with mortality. Of note, in the GUSTO-I database, CO was a better predictor of outcome than $\mathrm{CI}$, although the authors suggest that this may be related to the smaller number of patients with available CI data (10).

By receiver operator characteristic analysis, we were able to determine that a $\mathrm{CPO} \leq 0.53 \mathrm{~W}$ has a sensitivity and specificity of 0.66 to determine in-hospital mortality. The positive and negative predictive values for this CPO cutoff were $58 \%$ and $71 \%$, respectively. These relatively low predictive values - despite a strong association of CPO with mortality-reflect the strength of analyzing $\mathrm{CPO}$ as a continuous versus a categorical variable and demonstrate that there is not an important threshold effect. In a study examining the outcome of patients admitted due to acute heart failure and monitored by right heart catheterization, a similar cutoff point was found to be useful for predicting the rate of recurrent heart failure and death (G. Cotter, personal communication, April 15, 2003). The correlation between low CPO and poor outcome was demonstrated previously by Tan et al. (16), whose observed cutoff for increased mortality $(\mathrm{CPO}<1 \mathrm{~W})$ was, however, higher than the one determined in the present study. This discrepancy may be explained by the fact that in Tan's study (16) the CPO values for predicting prognosis were obtained at doses of pharmacologic support yielding the individual maximal $\mathrm{CPO}$, whereas in our study there was no prespecified protocol to record the $\mathrm{CPO}$ at maximal support. Furthermore, Tan et al. (16) did not use receiver operator characteristic analysis.

In addition to its prognostic significance, $\mathrm{CPO}$ can be determined without RHC. The measurement of $\mathrm{CO}$ by whole body electrical bioimpedance is accurate with the exception of diseases of the aorta and aortic valve and significant peripheral edema. It may also avoid the potential overestimation of $\mathrm{CO}$ by thermodilution in low $\mathrm{CO}$ states (14).

We found a significant decrease of CPI with increasing age. In normal aging hearts, there is a natural attrition of about 35\% of cardiac myocytes throughout adult life. Despite a compensatory cellular hypertrophic response, this loss of contractile cells leads to diminished cardiac function with decreased peak CPI and cardiac functional reserve $(33,34)$. Furthermore, the capacity of the cardiovascular system to react to a catecholamine-mediated response to stress may be diminished with advancing age (35-37).

Female gender was associated with a lower CPI, independent of age. Whether this is a result of differential catecholamine-mediated responsiveness or other mechanisms is unknown.

Left main culprit artery, three-vessel disease, and coronary perfusion pressure. Patients with left main MI appear to have higher right-sided pressures and SVR and lower CI and CPI than patients with other culprit arteries, but these differences did not reach statistical significance for most parameters, likely due to the small sample sizes.

Of note are the low median SVR values, despite vasopressor use, with a very wide range for all culprit arteries except for patients with left main MI. This might be related to an inflammatory component in the pathogenesis of cardiogenic shock (38).

In patients with left main MI, we found a lower coronary perfusion pressure than for all other culprit arteries. Coronary perfusion pressure was the only hemodynamic variable that was related to the number of diseased vessels. It was significantly lower in patients with three-vessel disease compared with patients with either zero/one-, or two-vessel disease. A decreased pressure gradient between the coronary arterial system and the LV plays a central role in the initiation and progression of the downward spiral of hypoperfusion and worsening LV dysfunction in cardiogenic shock (39). Below the lower limit of the autoregulatory range, coronary flow decreases markedly when the perfusion pressure is decreased (40). Thus, the high mortality for patients in cardiogenic shock with left main MI (78.6\%) and three-vessel disease $(50.8 \%$ vs. $39.8 \%$ vs. $35.0 \%$ for patients with two-vessel and zero/one-vessel disease, respectively, $\mathrm{p}$ $=0.002)$ is not surprising (41).

Study limitations. The majority of RHC measurements (73\%) were obtained while the patients had no IABP support. A total of $27 \%$ of patients had their RHC measurement completed in the time interval between IABP insertion and removal. Whether the IABP was on standby during the time of RHC measurement is not known. Intra-aortic balloon pump significantly increased mean arterial pressure and $\mathrm{CI}$ in a study of patients in cardiogenic shock (42). Therefore, IABP use is expected to increase CPO. Most patients were on supportive therapy with sympathomimetic amines (95\%) and/or dobutamine (60\%). These are likely to increase CPO, but the combination and intensity of various forms of support in relation to RHC measurements was not assessed. Cardiac 
power output remained a significant correlate of mortality after controlling for IABP use. It is unlikely that the predictive power of CPO was overestimated because of the supportive treatment. In contrast, $\mathrm{CPO}$ might be a stronger correlate of mortality in the absence of any support measures, because the difference in CPO between patients of various degrees of cardiovascular compromise may be blunted by the supportive therapy.

Conclusions. Cardiac power is a novel hemodynamic measure. By incorporating both flow and pressure domains, it represents the cardiac pumping ability. In the present study, cardiac power is the strongest hemodynamic correlate of outcome for patients in cardiogenic shock. Increasing age and female gender are independently associated with lower cardiac power.

\section{Acknowledgment}

The authors thank Dr. Lip Bun Tan for his valuable comments during the revision of the manuscript.

Reprint requests and correspondence: Dr. Judith S. Hochman, New York University School of Medicine, 530 First Avenue, HCC 1173, New York, New York 10016. E-mail: Judith. Hochman@med.nyu.edu.

\section{REFERENCES}

1. Connors AF Jr., Speroff T, Dawson NV, et al., for the SUPPORT Investigators. The effectiveness of right heart catheterization in the initial care of critically ill patients. JAMA 1996;276:889-97.

2. Dalen JE, Bone RC. Is it time to pull the pulmonary artery catheter? JAMA 1996;276:916-8.

3. Swan HJ, Forrester JS, Diamond G, Chatterjee K, Parmley WW. Hemodynamic spectrum of MI and cardiogenic shock: a conceptual model. Circulation 1972;45:1097-110.

4. Kaul S, Stratienko AA, Pollock SG, Marieb MA, Keller MW, Sabia PJ. Value of two dimensional echocardiography for determining the basis of hemodynamic compromise in critically ill patients: a prospective study. J Am Soc Echocardiogr 1994;7:598-606.

5. Menon V, Slater JN, White HD, Sleeper LA, Cocke T, Hochman JS. Acute myocardial infarction complicated by systemic hypoperfusion without hypotension: report of the SHOCK trial registry. Am J Med 2000;108:374-80.

6. Gottlieb S, Moss AJ, McDermott M, et al. Interrelation of left ventricular ejection fraction, pulmonary congestion and outcome in acute myocardial infarction. Am J Cardiol 1992;69:977-84.

7. Volpi A, De VC, Franzosi MG, et al. Determinants of six months mortality in survivors of myocardial infarction after thrombolysis: results of the GISSI-2 database. Circulation 1993;88:416-29.

8. Gore JM, Goldberg RJ, Spodick DH, Alpert JS, Dalen JE. A community wide assessment of the use of pulmonary artery catheters in patients with acute myocardial infarction. Chest 1987;92:721-7.

9. Zion MM, Balkin J, Rosenman D, et al., for the SPRINT Study Group. Use of pulmonary artery catheters in patients with acute myocardial infarction. Chest 1990;98:1331-5.

10. Hasdai D, Holmes DR, Califf RM, et al. Cardiogenic shock complicating acute myocardial infarction: predictors of death. Am Heart J 1999;138:21-31.

11. Hochman JS, Sleeper LA, Godfrey E, et al. Should we emergently revascularize occluded coronaries for cardiogenic shock: an international randomized trial of emergency PTCA/CABG-trial design. Am Heart J 1999;137:313-21.

12. Hochman JS, Sleeper LA, Webb JG, et al. Early revascularization in acute myocardial infarction complicated by cardiogenic shock. N Engl J Med 1999;341:625-34.
13. Cotter G, Williams SG, Vered Z, Tan LB. Role of cardiac power in heart failure. Curr Opin Cardiol 2003;18:215-22.

14. Cotter G, Moshkowitz Y, Kaluski E, et al. Accurate, noninvasive continuous monitoring of cardiac output by whole body electrical bio-impedance. Chest 2004;125:1431-40.

15. Cotter G, Moshkovitz Y, Kaluski E, et al. The role of cardiac power and vascular resistance in the pathophysiology, diagnosis and treatment of patients with congestive heart failure, pulmonary edema and cardiogenic shock. Eur J Heart Fail 2003;5:443-51.

16. Tan LB, Littler WA. Measurement of cardiac reserve in cardiogenic shock: implications for prognosis and management. Br Heart J 1990;64:121-8.

17. Cohen-Solal A, Tabet JY, Logeart D, Bourgoin P, Tokmakova M, Dahan M. A non-invasively determined surrogate of cardiac power ('circulatory power') at peak exercise is a powerful prognostic factor in chronic heart failure. Eur Heart J 2002;23:806-14.

18. Hochman JS, Buller CE, Sleeper LA, et al. Cardiogenic shock complicating acute myocardial infarction-etiologies, management and outcome: a report from the SHOCK trial registry. J Am Coll Cardiol 2000;36:1063-70.

19. Maruyama T, Lee JM, Nagano R, et al. Doppler echocardiographic pulmonary venous flow-velocity pattern for assessment of the hemodynamic profile in acute congestive heart failure. Am Heart J 1995; 129:107-13.

20. Picard MH, Davidoff R, Sleeper LA, et al. Echocardiographic predictors of survival and response to early revascularization in cardiogenic shock. Circulation 2003;107:279-84.

21. Menon V, Hochman JS, Stebbins A, et al. Lack of progress in cardiogenic shock: lessons from the GUSTO trials. Eur Heart J 2000;21:1928-36.

22. Bernard GR, Sopko G, Cerra F, et al. Pulmonary artery catheterization and clinical outcomes: National Heart, Lung, and Blood Institute and Food and Drug Administration workshop report. JAMA 2000; 283:2568-72.

23. The Vasodilation in the Management of Acute CHF (VMAC) Investigators. Intravenous nesiritide vs. nitroglycerin for the treatment of decompensated congestive heart failure. JAMA 2002;287:1531-40.

24. Sackner-Bernstein J, Kowalski M, Fox M. Is there risk associated with the use of nesiritide for acute heart failure. J Am Coll Cardiol 2003;41:161A.

25. Cuffe MS, Califf RM, Adams KF, et al. Short-term intravenous milrinone for acute exacerbation of chronic heart failure. JAMA 2002;287:1541-7.

26. Haas GJ, Young JB. Acute heart failure management. In: Topol EJ, editor. Textbook of Cardiovascular Medicine. 2nd ed. Philadelphia, PA: Lippincott Williams \& Wilkins, 2002:1856-8.

27. Williams SG, Cooke GA, Wright DJ, et al. Peak exercise cardiac power output; a direct indicator of cardiac function strongly predictive of prognosis in chronic heart failure. Eur Heart J 2001;22:1496-503.

28. Shah MR, Hasselblad V, Stinnett SS, et al. Dissociation between hemodynamic changes and symptom improvement in patients with advanced congestive heart failure. Eur J Heart Fail 2002;4:297-304.

29. Cotter G, Moshkovitz Y, Milovanov O, et al. Acute heart failure: a novel approach to its pathogenesis and treatment. Eur J Heart Fail 2002;4:227-34.

30. Morrow DA, Antman EM, Charlesworth A, et al. TIMI risk score for ST-elevation myocardial infarction: a convenient, bedside, clinical score for risk assessment at presentation. Circulation 2000;102:2031-7.

31. Normand SLT, Glickman ME, Sharma RGVRK, McNeil BJ. Using admission characteristics to predict short-term mortality from myocardial infarction in elderly patients. JAMA 1996;275:1322-8.

32. Lee KL, Woodlief LH, Topol EJ, et al. Predictors of 30-day mortality in the era of reperfusion for acute myocardial infarction. Circulation 1995;91:1659-68.

33. Olivetti G, Melissari M, Capasso JM, Anversa P. Cardiomyopathy of the aging human heart. Circ Res 1991;68:1560-8.

34. Goldspink DF, Burniston JG, Tan LB. Cardiomyocyte death and the aging and failing heart. Exp Physiol 2003;88:447-58.

35. Conway J, Wheeler R, Sannerstedt R. Symathetic nervous activity during exercise in relation to age. Cardiovasc Res 1971;5:577.

36. Julius S, Antoon A, Whitlock LS, Conway J. Influence of age on the hemodynamic response to exercise. Circulation 1967;36:222.

37. Lakatta EG, Levy D. Arterial and cardiac aging: major shareholders in cardiovascular disease enterprises. Part II: the aging heart in health: links to heart disease. Circulation 2003;107:346-54. 
38. Hochman JS. Cardiogenic shock complicating acute myocardial infarction: expanding the paradigm. Circulation 2003;107:2998-3002.

39. Hollenberg SM, Clifford JK, Parillo JE. Cardiogenic shock. Ann Intern Med 1999;131:47-59.

40. Hoffman JIE. Coronary physiology. In: Garfield OB, editor. Current Concepts in Cardiovascular Physiology. New York, NY: Academic Press, 1990:289-349.

41. Wong SC, Sanborn T, Sleeper LA, et al. Angiographic findings and clinical correlates in patients with cardiogenic shock complicating acute myocardial infarction: a report from the SHOCK trial registry. J Am Coll Cardiol 2000;36:1077-83.

42. Mueller H, Ayres SM, Gianelli S, Conklin EF, Mazzara JT, Grace WJ. Effect of isoproterenol, 1-norepinephrine, and intraaortic counterpulsation on hemodynamics and myocardial metabolism in shock following acute myocardial infarction. Circulation 1972;45: 335-51. 